\title{
Analysis of Transverse Vibration Acceleration for a High-speed Elevator with Random Parameter Based on Perturbation Theory
}

\author{
Chen Wang, Ruijun Zhang and Qing Zhang \\ College of Mechanical and Electronic Engineering, Shangdong Jianzhu University, Jinan(250101), China.
}

\begin{abstract}
(Received 24 September 2015; accepted 4 September 2016)
The problem of randomness of design parameters objectively exists in the high-speed elevator cabin system. The definite and random part of the acceleration response expressions were derived according to perturbation theory and the transverse vibration acceleration response of the observation point was analyzed. The sensitivity expressions of various random parameters to the acceleration response were deduced by solving the random response of expression and comparing the coefficients of various parameters of the random part. The different impacts of various parameters on the acceleration response were analyzed according to the expression of acceleration response sensitivity. Based on the displacement response covariance matrix and random parameter covariance matrix, the standard deviation characteristic of the acceleration response was obtained and analyzed. The results showed that the random parameters made the acceleration response to be more discrete. Additionally, the randomness of the geometrical parameters had the greatest influence on transverse acceleration. The results can provide a reference for anti-vibration design of high-speed elevators and safety assessment.
\end{abstract}

\section{INTRODUCTION}

As a means of transport in high-rise buildings, the elevator's speed was rapidly increased with the increase of the height of buildings. ${ }^{1}$ Currently, the elevator whose running speed is more than $2.5 \mathrm{~m} / \mathrm{s}$ is known as the high-speed elevator. With the increase of elevator's speed, the transverse vibration problem due to rail harshness has become more and more serious. In recent years, many domestic and foreign scholars have studied the vibration of the cabin system. Noguchi et al. used frequency-domain analysis based on principal component analysis to perform operational modal analysis of an elevator car. $^{2}$ Yin et al. transformed many important factors such as rail harshness, flexion of the rail, and the guide shoe's defects into the contact force of rail to guide shoe, and established a multi-DOF transverse vibration model of a high-speed elevator car. ${ }^{3} \mathrm{Xia}$ and Shi established a transverse vibration model of an elevator car by studying real-time interface stiffness between the guide rollers and the guide shoe. ${ }^{4}$ They took rail harshness as the input excitation to study the transverse vibration of an elevator car. In the product design stage, all of the parameters are definite. However, due to the influence of actual installation circumstance, live debugging, manufacturing errors, installation errors, the influence of temperature and uncertainty of physical parameters of materials, etc., the parameters of the same batch of elevator products are different. For an actual product, its parameters are uncertain and present some randomness. For example, Li and Liao analyzed a shear wall's vibration response..$^{5}$ In the case of the variation, the coefficient of elasticity modulus was 0.3 and the vibration power spectral density of the top of the shear wall was increasing exponentially. However, most of the existing literature about the elevator car's vibration does not consider the random parameters of an elevator car or approximate them as definite parameters. In fact, these random parameters not only affect the eigenvalues and eigenvectors of the system in each mode, but also have an effect on the statistical properties of the response. Therefore, studying the response to random parameters is significant to the structure of elevator car vibration damping, reliability sensitivity analysis, and safety assessment.

Zhang et al. studied the frequency response function of a statistical feature of one and two degrees of freedom random structure using the Monte-Carlo method. ${ }^{6}$ Zhang et al. used the stochastic finite element method to study reliability characteristics of a mechanical structure component under complex loading conditions. ${ }^{7}$ The Monte-Carlo numerical modeling method is highly accuracy, but for large and complex structures, it is time consuming. Thus, it is suitable for the comparison of methods but not suitable for practical engineering structural analysis. The stochastic finite element method needs to set up all kinds of random parameters corresponding to the stochastic finite element characteristic matrix, which causes much inconvenience to its computer program design. Therefore, it is necessary to find a random perturbation method to avoid the establishment of a random finite element characteristic matrix which can be realized easily via computation.

\section{CONSTRUCTION OF A RANDOM PARAMETER CAR VIBRATION MODEL}

The establishment of the car model is the basis for solving the differential equations of the motion of car system. The model should not be too simple, for it could not accurately reflect the actual situation. ${ }^{8}$ On the other hand, the model cannot be too complicated because the amount of calculations will be too large or even impossible to solve. In Fig. 1, a car vibration model is established. A rectangular coordinate system oxyz is established with the barycenter of the car as the origin. The roller guide shoe system is simplified into a spring damping system. ${ }^{9,10}$ Each system provides the $x$ and $y$ directions of the two forces, and so the whole system of the car has 5 DOF along the $x$ and $y$ directions and around the $x, y$ and $z$-axis rotations. $^{11}$

For convenient calculation, suppose that the horizontal distances from guide wheel 1, 2 and guide wheel 3, 4, to the cen- 


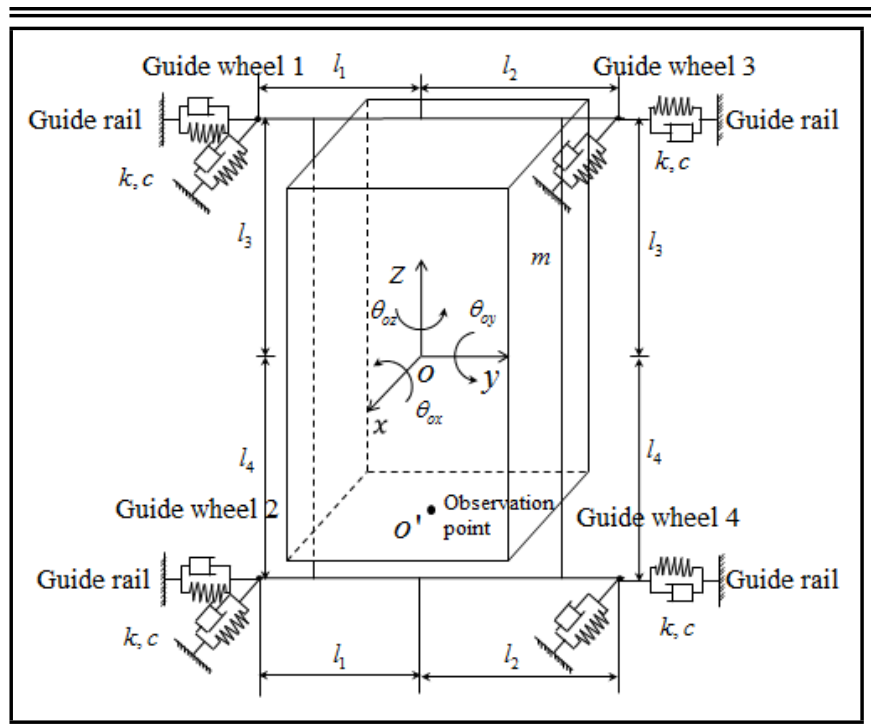

Figure 1. Parameters of elevator cabin system.

troid are respectively equal to $l_{1}$ and $l_{2}$. While the vertical distances from guide wheel 1, 3 and guide wheel 2, 4, to the centroid are equal to $l_{3}$ and $l_{4}$. As described above, the system has 10 random parameters: the mass of the car $m$, the $x$-axis rotational moment of inertia $J_{o x}$, the $y$-axis rotational moment of inertia $J_{o y}$, the $z$-axis rotational moment of inertia $J_{o z}$, stiffness and damping in all directions of the guide wheel $k, c$ (assume that the guide wheels' stiffness and damping at all directions are equal), $l_{1}, l_{2}, l_{3}$, and $l_{4}$. Additionally, they are assumed to be independent with each other. And they have their own mean value and standard deviation. In the following, the dynamic response of the point of observation point $O^{\prime}$ at the bottom of the car was studied.

The elevator car system dynamics equation can be expressed as: ${ }^{12}$

$$
M \ddot{X}+C \dot{X}+K X=P(t)
$$

where $M, C$, and $K$ are the mass matrix, damping matrix, and stiffness matrix respectively, $X$ is the generalized coordinate array, and $P(t)$ is an external stimulus. $M=\left[\begin{array}{ccccc}m & 0 & 0 & 0 & 0 \\ 0 & m & 0 & 0 & 0 \\ 0 & 0 & J_{o x} & 0 & 0 \\ 0 & 0 & 0 & J_{o y} & 0 \\ 0 & 0 & 0 & 0 & J_{o z}\end{array}\right]$,

$C=\sum_{i=1}^{4}\left[\begin{array}{ccccc}c_{x i} & 0 & 0 & c_{x i} l_{z i} & -c_{x i} l_{y i} \\ 0 & c_{y i} & -c_{y i} l_{z i} & 0 & 0 \\ 0 & -c_{y i} l_{z i} & c_{y i} l_{z i}^{2} & 0 & 0 \\ c_{x i} l_{z i} & 0 & 0 & c_{x i} l_{z i}^{2} & -c_{x i} l_{y i} l_{z i} \\ -c_{x i} l_{y i} & 0 & 0 & -c_{x i} l_{y i} l_{z i} & c_{x i} l_{y i}^{2}\end{array}\right]$,
$K=\sum_{i=1}^{4}\left[\begin{array}{ccccc}k_{x i} & 0 & 0 & k_{x i} l_{z i} & -k_{x i} l_{y i} \\ 0 & k_{y i} & -k_{y i} l_{z i} & 0 & 0 \\ 0 & -k_{y i} l_{z i} & k_{y i} l_{z i}^{2} & 0 & 0 \\ k_{x i} l_{z i} & 0 & 0 & k_{x i} l_{z i}^{2} & -k_{x i} l_{y i} l_{z i} \\ -k_{x i} l_{y i} & 0 & 0 & -k_{x i} l_{y i} l_{z i} & k_{x i} l_{y i}^{2}\end{array}\right]$,

$X=\left[\begin{array}{c}x \\ y \\ \theta_{o x} \\ \theta_{o y} \\ \theta_{o z}\end{array}\right]$

\section{DYNAMIC RESPONSE ANALYSIS OF THE RANDOM PARAMETERS OF THE ELEVATOR CAR SYSTEM}

In this section, the dynamic response of the car system was discussed by using the random perturbation method. ${ }^{13}$ Since $M, C, K$, and $P(t)$ have random properties, the following transformations are needed:

$$
\begin{gathered}
M=M_{d}+\varepsilon M_{r} ; \\
C=C_{d}+\varepsilon C_{r} ; \\
K=K_{d}+\varepsilon K_{r} ; \\
X=X_{d}+\varepsilon X_{r} ; \\
P(t)=P_{d}(t)+\varepsilon P_{r}(t) ;
\end{gathered}
$$

where $\varepsilon$ is a small parameter. As an abstract value obtained by the perturbation method will not affect the derivation of the entire equation. ${ }^{14}$ The subscript $d$ and subscript $r$ denote the determining parts and random parts of random parameters, and the mean of the random parts is zero. By combining these equations with Eq. (1), comparing them with factor $\varepsilon$ at the same power, and omitting higher-order terms above $O\left(\varepsilon^{2}\right)$, the following equations were obtained: ${ }^{15}$

$$
\varepsilon^{0}: M_{d} \ddot{X}_{d}+C_{d} \dot{X}_{d}+K_{d} X_{d}=P_{d}(t)
$$

$$
\begin{aligned}
\varepsilon^{1}: M_{d} \ddot{X}_{r}+C_{d} \dot{X}_{r}+K_{d} X_{r}= & \\
& P_{r}(t)-\left(M_{r} \ddot{X}_{d}+C_{r} \dot{X}_{d}+K_{r} X_{d}\right) .
\end{aligned}
$$

In order to calculate the random response generated due to random external excitation and random parameters, the random response was written as:

$$
X_{r}=X_{r 1}+X_{r 2}
$$

where $X_{r 1}$ and $X_{r 2}$ satisfy the following equations:

$$
M_{d} X_{r 1}+C_{d} X_{r 1}+K_{d} X_{r 1}=P_{r}(t)
$$

$$
\begin{aligned}
M_{d} \ddot{X}_{r 2}+C_{d} \dot{X}_{r 2}+ & K_{d} X_{r 2}= \\
& -\left(M_{r} \ddot{X}_{d}+C_{r} \dot{X}_{d}+K_{r} X_{d}\right)
\end{aligned}
$$

Equation (10) and Eq. (11) respectively denote the random response due to the randomness of the excitation and of the random parameters. For Eq. (11), random variables were expanded into the Taylor series in the vicinity of determining part $b_{d j}(j=1,2, \ldots, m)$ of the random parameters, and substituted into Eq. (11), and comparing the $b_{r j}$ coefficients the following equation was obtained: ${ }^{16,17}$

$$
\begin{aligned}
M_{d} \frac{\partial \ddot{X}_{d}}{\partial b_{j}}+ & C_{d} \frac{\partial \dot{X}_{d}}{\partial b_{j}}+K_{d} \frac{\partial X_{d}}{\partial b_{j}}= \\
& -\left(\frac{\partial M_{d}}{\partial b_{j}} \ddot{X}_{d}+\frac{\partial C_{d}}{\partial b_{j}} \dot{X}_{d}+\frac{\partial K_{d}}{\partial b_{j}} X_{d}\right) ; \\
& (j=1,2, \ldots, m) .
\end{aligned}
$$


Equation (12) was solved by using the Wilson- $\theta$ method. ${ }^{18}$ And the system response sensitivities were obtained as: $\frac{\partial \ddot{X}_{d}}{\partial b_{j}}$, $\frac{\partial \dot{X}_{d}}{\partial b_{j}}, \frac{\partial X_{d}}{\partial b_{j}}, \ddot{X}_{r 2} \dot{X}_{r 2} X_{r 2}$. They were expanded into the Taylor series in the vicinity of determining part $b_{d j}(j=1,2, \ldots, m)$ of the random parameters and the following equations were obtained:

$$
\begin{aligned}
& \ddot{X}_{r 2}=\sum_{j=1}^{m} \frac{\partial \ddot{X}_{d}}{\partial b_{j}} \cdot b_{r j} ; \\
& \dot{X}_{r 2}=\sum_{j=1}^{m} \frac{\partial \dot{X}_{d}}{\partial b_{j}} \cdot b_{r j} \\
& X_{r 2}=\sum_{j=1}^{m} \frac{\partial X_{d}}{\partial b_{j}} \cdot b_{r j}
\end{aligned}
$$

Thus, the random responses of the random parameter structure $\ddot{X}_{r 2}, \dot{X}_{r 2}$, and $X_{r 2}$ were obtained. The observation point $O^{\prime}$ in the $x$-direction and $y$-direction acceleration of the matrix is:

$$
\left[\begin{array}{c}
\ddot{x}_{o^{\prime}} \\
\ddot{y}_{o^{\prime}}
\end{array}\right]=T \ddot{X}
$$

where $T$ is the transformation matrix: $T=$ $\left[\begin{array}{ccccc}1 & 0 & 0 & l_{z o^{\prime}} & l_{y o^{\prime}} \\ 0 & 1 & l_{z o^{\prime}} & 0 & 0\end{array}\right]$

\section{STANDARD DEVIATION ANALYSIS FOR THE DYNAMIC RESPONSE OF THE ELEVATOR CAR}

In consideration of the complexity and difficulty of the randomness of the external excitation of the car, only the standard deviation of the random response due to random $X_{r 2}$ parameter was studied to facilitate random vibration system optimization.

Firstly, the displacement response covariance matrix $N_{x}$, random parameter covariance matrix $N_{b}$, and displacement response sensitivity matrix $\left[\frac{\partial X_{d}}{\partial b}\right]$ were defined as:

$$
\begin{aligned}
& N_{x}= \\
& {\left[\begin{array}{cccc}
\operatorname{Var}\left(X^{(1)}\right) & \operatorname{Cov}\left(X^{(2)}, X^{(1)}\right) & \cdots & \operatorname{Cov}\left(X^{(k)}, X^{(1)}\right) \\
\operatorname{Cov}\left(X^{(2)}, X^{(1)}\right) & \operatorname{Var}\left(X^{(1)}\right) & \cdots & \operatorname{Cov}\left(X^{(k)}, X^{(2)}\right) \\
\vdots & \vdots & \ddots & \vdots \\
\operatorname{Cov}\left(X^{(k)}, X^{(1)}\right) & \operatorname{Cov}\left(X^{(k)}, X^{(2)}\right) & \cdots & \operatorname{Var}\left(X^{(k)}\right)
\end{array}\right] ;}
\end{aligned}
$$

$$
N_{b}=\left[\begin{array}{cccc}
\operatorname{Var}\left(b_{1}\right) & \operatorname{Cov}\left(b_{2}, b_{1}\right) & \ldots & \operatorname{Cov}\left(b_{m}, b_{1}\right) \\
\operatorname{Cov}\left(b_{2}, b_{1}\right) & \operatorname{Var}\left(b_{2}\right) & \ldots & \operatorname{Cov}\left(b_{m}, b_{2}\right) \\
\vdots & \vdots & \ddots & \vdots \\
\operatorname{Cov}\left(b_{m}, b_{1}\right) & \operatorname{Cov}\left(b_{m}, b_{2}\right) & \cdots & \operatorname{Var}\left(b_{m}\right)
\end{array}\right]_{(18)}
$$$$
\left[\begin{array}{c}
\frac{\partial X_{d}}{\partial b}
\end{array}\right]=\left[\begin{array}{llll}
\frac{\partial X_{d}}{\partial b_{1}} & \frac{\partial X_{d}}{\partial b_{2}} & \cdots & \frac{\partial X_{d}}{\partial b_{m}}
\end{array}\right] .
$$

Table 1. Parameters of elevator cabin system.

\begin{tabular}{||c|c|c|c|c|c||}
\hline $\begin{array}{c}\text { Parameter: } \\
b_{j}\end{array}$ & $\begin{array}{c}\text { Mean } \\
\text { value: } \\
\mathrm{D} b_{j}\end{array}$ & $\begin{array}{c}\text { Standard } \\
\text { deviation: } \\
\sigma_{b_{j}} \\
(\mathrm{CV}=0.05)\end{array}$ & $\begin{array}{c}\text { Para- } \\
\text { meter: } \\
b_{j}\end{array}$ & $\begin{array}{c}\text { Mean } \\
\text { value: } \\
\mathrm{D} b_{j}\end{array}$ & $\begin{array}{c}\text { Standard } \\
\text { deviation: } \\
\sigma_{b_{j}} \\
(\mathrm{CV}=0.05)\end{array}$ \\
\hline$m[\mathrm{~kg}]$ & 1000 & 50 & $c[\mathrm{~kg} / \mathrm{s}]$ & 1000 & 50 \\
\hline$J_{o x}\left[\mathrm{~kg} \cdot \mathrm{m}^{2}\right]$ & 2300 & 115 & $l_{1}[\mathrm{~m}]$ & 1 & 0.05 \\
\hline$J_{o y}\left[\mathrm{~kg} \cdot \mathrm{m}^{2}\right]$ & 2000 & 100 & $l_{2}[\mathrm{~m}]$ & 1 & 0.05 \\
\hline$J_{O z}\left[\mathrm{~kg} \cdot \mathrm{m}^{2}\right]$ & 820 & 41 & $l_{3}[\mathrm{~m}]$ & 1.6 & 0.08 \\
\hline$k\left[\mathrm{~N} \cdot \mathrm{m}^{-1}\right]$ & 100000 & 5000 & $l_{4}[\mathrm{~m}]$ & 1.4 & 0.07 \\
\hline
\end{tabular}

$\operatorname{Var}\left(X^{(k)}\right)$ represents the variance of the $k_{t h}$ element in vector $X$, and Cov represents covariance. The following equation was obtained:

$$
N_{x}=\left[\frac{\partial X_{d}}{\partial b}\right] N_{b}\left[\frac{\partial X_{d}}{\partial b}\right]^{T} .
$$

Based on Eq. (20), the standard deviation of the displacement response was obtained

$$
\sigma_{x}^{i}=\left(\sum_{j=1}^{m} \sum_{k=1}^{m} \frac{\partial X_{d}^{i}}{\partial b_{j}} \frac{\partial X_{d}^{i}}{\partial b_{k}} \sigma_{b j} \sigma_{b k} \rho_{j k}\right)^{1 / 2}
$$

where $\sigma_{x}^{i}$ is the standard deviation $\left[\operatorname{Var}\left(x^{(i)}\right)\right]^{1 / 2}$ of the $\mathbf{i}_{\text {th }}$ element in vector $X, \rho_{j k}$ is the correlation coefficient of $b_{j}$ and $b_{k}$, and $\sigma_{b j}$ is the standard deviation of $b_{j}$.

Similarly, the standard deviation of the velocity response and acceleration response were obtained:

$$
\begin{aligned}
\sigma_{\dot{x}}^{i} & =\left(\sum_{j=1}^{m} \sum_{k=1}^{m} \frac{\partial \dot{X}_{d}^{i}}{\partial b_{j}} \frac{\partial \dot{X}_{d}^{i}}{\partial b_{k}} \sigma_{b j} \sigma_{b k} \rho_{j k}\right)^{1 / 2} ; \\
\sigma_{\ddot{x}}^{i} & =\left(\sum_{j=1}^{m} \sum_{k=1}^{m} \frac{\partial \ddot{X}_{d}^{i}}{\partial b_{j}} \frac{\partial \ddot{X}_{d}^{i}}{\partial b_{k}} \sigma_{b j} \sigma_{b k} \rho_{j k}\right)^{1 / 2} .
\end{aligned}
$$

It can be seen that as long as the standard deviation of the system architecture's random parameters and correlation coefficients are given, the standard deviation of the response can be obtained without involving the statistical properties of other random parameters. This makes it easy to be applied it to engineering practice. Besides, only the required degree of freedom is used to calculate the response sensitivity to avoid a large amount of computation.

\section{CASE ANALYSIS OF AN ELEVATOR CAR SYSTEM}

\subsection{Calculation of the Acceleration Response of a Random Parameters Elevator Car System}

According to Table 1, the elevator car system vibration model includes the mass parameters $\mathrm{m}, J_{o x}, J_{o y}$, and $J_{o z}$; the dynamical parameters $k$ and $c$; and the geometrical parameters $l_{1}, l_{2}, l_{3}$, and $l_{4}$. It is assumed that the random parameters are independent and subject to standard normal distribution. For a type of high-speed traction elevator with a running speed of $5 \mathrm{~m} / \mathrm{s}$, the parameters are shown in Table 1.

It is considered that the parameters are independent and subject to the standard normal distribution and the coefficient of 


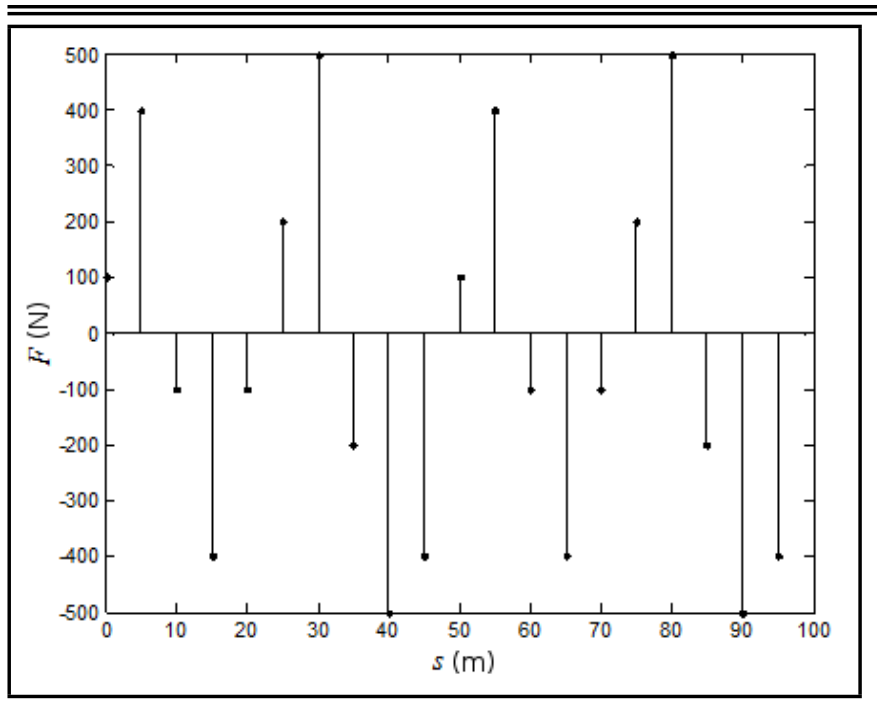

Figure 2. Lead rail's excitation in the $x$-direction.

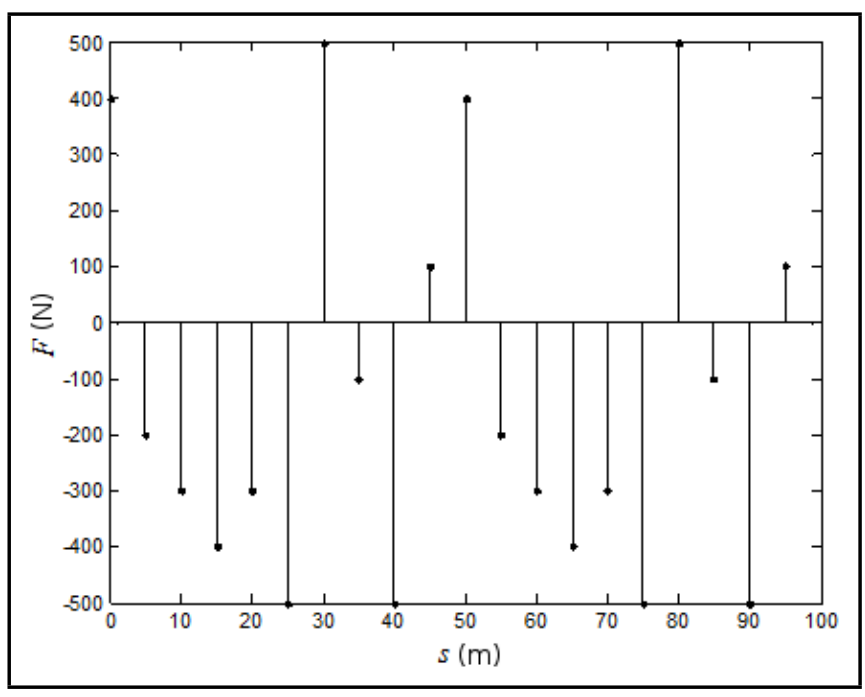

Figure 3. Lead rail's excitation in the $y$-direction.

variation $\mathrm{CV}$ is fixed at 0.05 . For convenient calculation, the step pulse excitation was applied to the $x$ and $y$ directions in the left side of the rail joints and the right side of the rail was regarded as the ideal rail. A single rail length was $5 \mathrm{~m}$. The excitation was shown in Fig. 2 and Fig. 3.

The response expression was constructed by perturbation theory and the response acceleration $\ddot{X}_{d}$ was obtained by using the Wilson- $\theta$ Method to solve Eq. (7). Then, the $x$-direction and the $y$-direction accelerations of any point were obtained by letting the acceleration response pre-multiply the transformation matrix $T$. Images of response acceleration of determining parts in the $x$-direction and $y$-direction were obtained from the center point of the bottom of the elevator car, as shown in Fig. 4 and Fig. 5. As can be seen from the figures, the transverse vibration in the $\mathrm{x}$ direction tended to be relatively unstable at any excitation, while the vibration intensity in the $y$ direction was generally greater than that in the $x$ direction.

The random part of the acceleration response was obtained by letting the $b r j$ equal to $\pm \sigma_{b j}$ together with the $\ddot{X}_{d}$ substituting it into Eq. (13) and solving it. Then, the total acceleration response $\ddot{X}$ was obtained by combining the random part and the definite part of the acceleration response and multiplying $\ddot{X}$ by transformation matrix $T$ and then obtained the observation point total acceleration response, as shown in Fig. 6 and

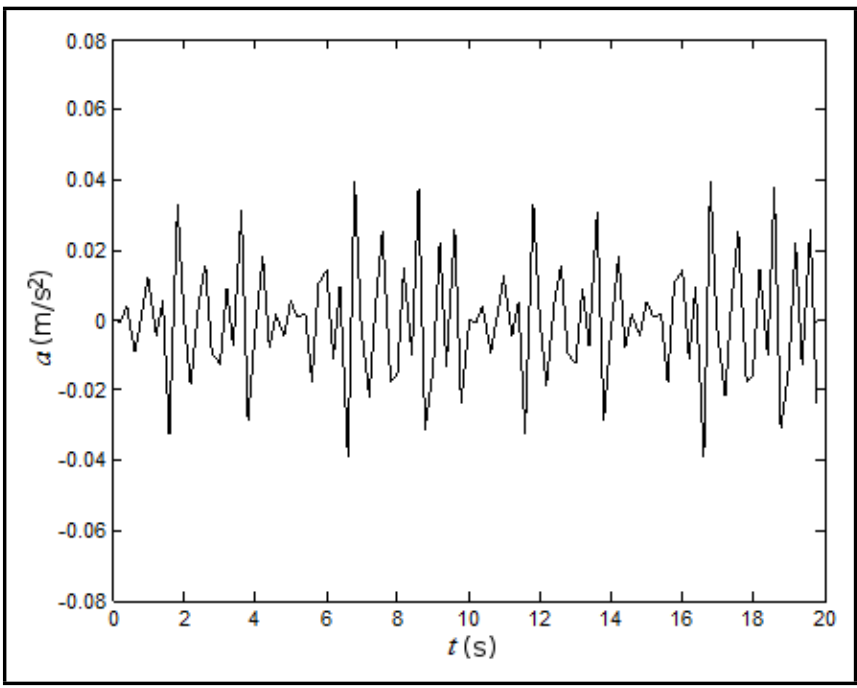

Figure 4. Certain part's acceleration of the observation point in the $x$ direction.

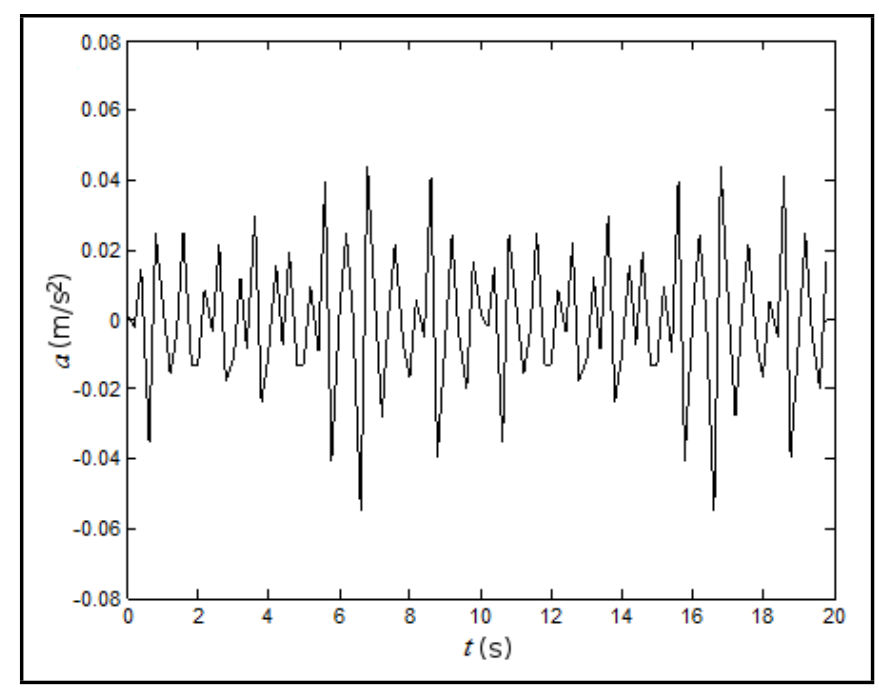

Figure 5. Certain part's acceleration of the observation point in the $y$ direction.

Fig. 7.

Taking the random parameters into consideration, it can be seen that the absolute value of the maximum accelerations in the $x$ direction and the $y$ direction were increased by about $50 \%$ and $40 \%$ by comparing acceleration response determining parts. Furthermore, the response value of each moment had a different degree of dispersion by comparing the determining part. Especially at $6.5 \mathrm{~s}$ and $16.5 \mathrm{~s}$, the $y$ direction always showed more abrupt acceleration response characteristics. By considering the randomness of the structural parameters, the discrete degree of acceleration response was indeed increased.

\subsection{Acceleration Response Analysis}

The stochastic parameter system response sensitivity Eq. (12) was solved by the Wilson- $\theta$ method, and the acceleration response sensitivity for each random parameter in the $\mathrm{x}$ direction and $\mathrm{y}$ direction at the observation point (the $\frac{\partial \ddot{X}_{x}}{b_{j}}$ and $\frac{\partial \ddot{X}_{y}}{b_{j}}$ ) can be obtained. The acceleration response sensitivities $\frac{\partial \ddot{X}_{x}}{b_{j}}$ and $\frac{\partial \ddot{X}_{y}}{b_{j}}$ were constantly changing in the whole time period. In order to facilitate the comparison, the mean 


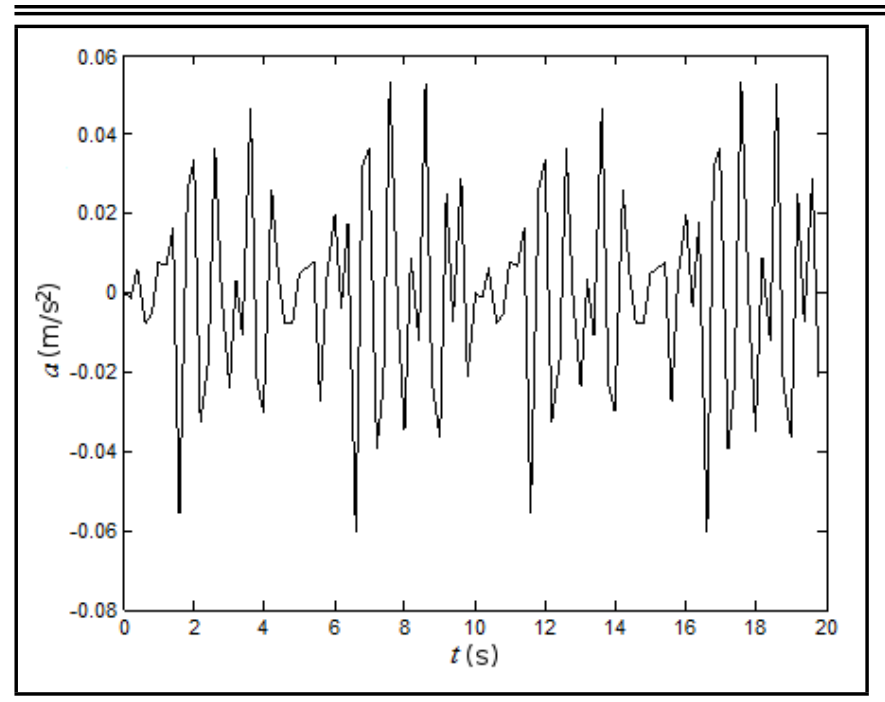

Figure 6. General acceleration of the observation point in the $x$-direction.

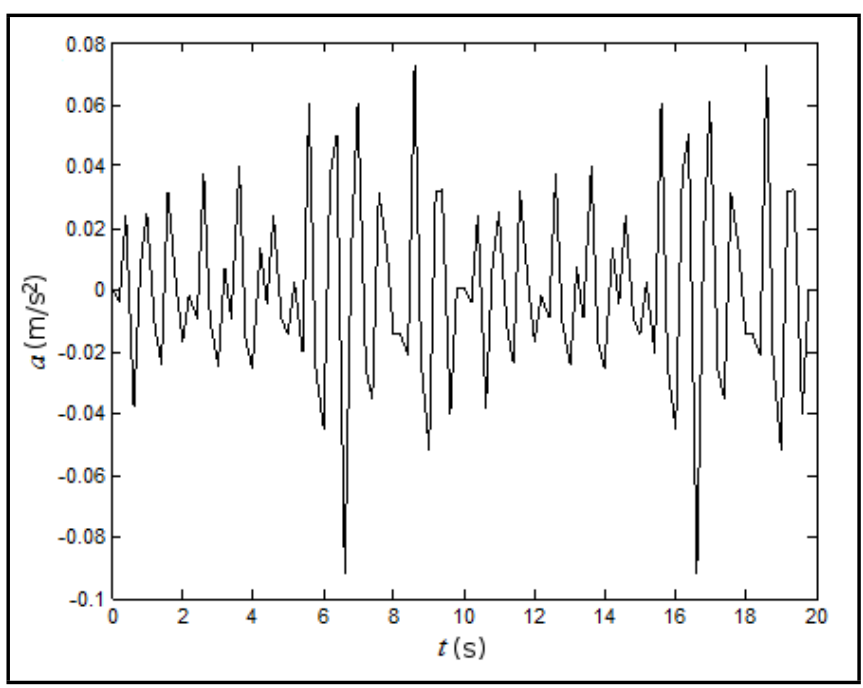

Figure 7. General acceleration of the observation point in the $y$-direction.

acceleration response sensitivities $E\left|\frac{\partial \ddot{X}_{x}}{b_{j}}\right|$ and $E\left|\frac{\partial \ddot{X}_{y}}{b_{j}}\right|$ were obtained by calculating the mean of absolute value of the $\frac{\partial \ddot{X}_{x}}{b_{j}}$ and $\frac{\partial \ddot{X}_{y}}{b_{j}}$. The results were shown in Table 2 .

As can be seen from the table, the acceleration response sensitivity in the $y$ direction of the observation point was generally larger than that in the $x$ direction, which indicates that the influence of the change of the random parameters on the acceleration response in the $y$ direction was larger. In addition, the effects from the mess parameters $m, J_{o x}, J_{o y}$, and $J_{o z}$, and dynamical parameters $k$ and $c$ on the sensitivity of the response were relative low. Thus, they can be used as the determination of the parameters, while the geometrical parameters $l_{1}, l_{2}$, $l_{3}$, and $l_{4}$ had a higher impact on the response sensitivity, and should be treated as random parameter.

\subsection{Mean and Standard Deviation Analysis of Observation Point}

In this case, the guide rail excitation is deterministic. The acceleration response's standard deviations (SD) of the observation point in the $x$ direction and $y$ direction at the first second were calculated, then SDs of the acceleration response were divided by the acceleration mean value (MV) based on pertur- bation theory to obtain the coefficient of variation $(\mathrm{CV})$. The results were shown in Table 3.

From the table, the variation coefficient of the acceleration response were generally around 1 in the case of the random parameters of the variation coefficient $\mathrm{CV}$ equal to 0.05 . Moreover, after comparing the determine part of the acceleration and the total acceleration image at the observation point, it can be shown that the actual response of the discrete degree was large.

In conclusion, the randomness of the design parameters of the elevator car system had an obvious effect on the acceleration of the center of the elevator car.

\section{CONCLUSIONS}

In this paper, an elevator car vibration model with random parameters was established, and the dynamic response expressions for determining random parts of an arbitrary point were established by using stochastic perturbation theory. The calculation results showed that the randomness of the structural parameters had a certain effect on the response.

Response sensitivity expression can be applied in solving the transverse acceleration response's sensitivity of each random parameter to the observation point. The acceleration sensitivity of geometrical parameters was much higher than the mass and dynamic parameters. For convenient calculation, the random parameters with a relative lower acceleration response sensitivity were simplified as the determining parameters. In the manufacturing and installation processes the parameters with higher sensitivity should be strictly controlled.

Based on the analysis of the response characteristics of the acceleration, the random parameters caused the coefficient of variation of the acceleration response becoming larger, which further confirms that the random parameters have a greater impact on the response. The random parameters cannot be simplified to the determining parameters.

\section{ACKNOWLEDGEMENTS}

Authors are grateful for the financial support by Shandong Jianzhu University Doctor Foundation (No.XNBS1514).

\section{REFERENCES}

1 Wang, W. and Qian, J. Transverse vibration analysis for an elevator suspended system subjected to displacement excitation due to structure sway, Journal of Vibration And Shock, 32 (7), 70-73, (2013). https://dx.doi.org/10.13465/j.cnki.jvs.2013.07.033

2 Naoaki, N., Atsushi, A., Koichi, M.,et al. Study on active vibration control for high-speed elevators, Journal of System Design and Dynamics, 5 (1), 164-179, (2011). https://dx.doi.org/10.1299/jsdd.5.164

3 Yin Ji-cai, Bing Yan-nian, Jiang Li-ming, et al. Research on high-speed elevator MDOF horizontal dynamic characteristics and simulation, Journal of Machine Design, 28 (10), 70-73, (2011). https://dx.doi.org/10.13841/j.cnki.jxsj.2011.10.022

4 Xia Bing-hu and Shi Xi. Horizontal vibrations of high-speed elevator with guide rail excitation, $M a$ chine Building and Automation, 41 (5), 161-165, (2012). https://dx.doi.org/10.3969/j.issn.1671-5276.2012.05.052 
Table 2. Acceleration response sensitivity of random parameters.

\begin{tabular}{||c|c|c|c|c|c||}
\hline \multirow{2}{*}{ Sensitivity } & \multicolumn{5}{|c|}{ Parameters } \\
\cline { 2 - 6 } & $b_{1}(m)$ & $b_{2}\left(J_{o x}\right)$ & $b_{3}\left(J_{o y}\right)$ & $b_{4}\left(J_{o z}\right)$ & $b_{5}(k)$ \\
\hline$E\left|\frac{\partial \ddot{X}_{x}}{b_{j}}\right|$ & $1.01 \times 10^{-4}$ & 0 & $4.38 \times 10^{-5}$ & 0 & $1.94 \times 10^{-5}$ \\
\hline$E\left|\frac{\partial \ddot{X}_{y}}{b_{j}}\right|$ & $1.11 \times 10^{-4}$ & $5.42 \times 10^{-5}$ & 0 & 0 & $2.84 \times 10^{-5}$ \\
\hline \hline$E\left|\frac{\partial \ddot{X}_{x}}{b_{j}}\right|$ & $2.67 \times 10^{-6}$ & $1.17 \times 10^{-1}$ & $1.17 \times 10^{-1}$ & $2.36 \times 10^{-1}$ & $3.40 \times 10^{-1}$ \\
\hline$E\left|\frac{\partial \ddot{X}_{y}}{b_{j}}\right|$ & $3.72 \times 10^{-6}$ & 0 & 0 & $2.44 \times 10^{-1}$ & $4.49 \times 10^{-1}$ \\
\hline
\end{tabular}

Table 3. Acceleration response sensitivity of random parameters.

\begin{tabular}{|c|c|c|c|c|c|c|}
\hline \multicolumn{2}{|c|}{ Time $[\mathrm{s}]$} & 0.2 & 0.4 & 0.6 & 0.8 & 1.0 \\
\hline \multirow{2}{*}{$\mathrm{MV}(\mathrm{CV})$} & X Direction & $\begin{array}{c}0.0037 \\
(1.5135)\end{array}$ & $\begin{array}{c}-0.0064 \\
(1.3125)\end{array}$ & $\begin{array}{c}0.0380 \\
(0.7052)\end{array}$ & $\begin{array}{c}-0.0905 \\
(0.7292)\end{array}$ & $\begin{array}{c}0.0106 \\
(4.5094)\end{array}$ \\
\hline & Y Direction & $\begin{array}{c}0.0124 \\
(0.7661)\end{array}$ & $\begin{array}{c}-0.0190 \\
(1.8684)\end{array}$ & $\begin{array}{c}0.1481 \\
(0.7353)\end{array}$ & $\begin{array}{c}-0.3555 \\
(0.2472)\end{array}$ & $\begin{array}{c}0.2438 \\
(0.5947)\end{array}$ \\
\hline \multirow{2}{*}{ SD } & X Direction & 0.0056 & 0.0084 & 0.0268 & 0.0660 & 0.0478 \\
\hline & Y Direction & 0.0095 & 0.0355 & 0.1089 & 0.0879 & 0.1450 \\
\hline
\end{tabular}

5 Jie Li and Songtao Liao. Response analysis of stochastic parameter structures under non-stationary random excitation, Computational Mechanics, 27 (1), 61-68, (2001). https://dx.doi.org/10.1007/s004660000214

${ }^{6}$ Zhang Qiang, Shi Feng-ying, and Liu Jian-xin. Analysis of frequency response function of structures with random parameters. Journal of Vibration and Shock, 25 (2), 64-66, (2006). https://dx.doi.org/10.3969/j.issn.10003835.2006.02.017

${ }^{7}$ Zhang Hong-qi, Shao Xiao-dong, Hu Xiang-tao, et al. Reliability assessment for mechanical structure based on stochastic finite element method, Journal of Southeast University (Natural Science Edition), 44 (1), 76-81, (2014). https://dx.doi.org/10.3969/j.issn.1001-0505.2014.01.014

8 Arrasate, X., Kaczmarczyk, S., Almandoz, G., et al. The modelling, simulation and experimental testing of the dynamic responses of an elevator system, Mechanical Systems and Signal Processing, 42 (1), 258-282, (2014). https://dx.doi.org/10.1016/j.ymssp.2013.05.021

${ }^{9}$ Mei De-qing, Du Xiao-qiang, and Chen Zi-chen. Vibration analysis of high-speed traction elevator based on guide roller-railcontact model, Journal of Mechanical Engineering, 45 (5), 264-270, (2009). https://dx.doi.org/10.3901/JME.2009.05.264

${ }^{10}$ Du Xiao-qiang, Mei De-qing, and Chen Zi-chen. Timevarying element model of high-speed tractiong elevator and its horizontal vibration response analysis, Journal of Zhejiang University(Engineering Science), 43 (1), 148-152, (2009). https://dx.doi.org/10.3785/j.issn.1008973X.2009.01.02

${ }^{11}$ Feng Yong-hui and Zhang Jian-wu. The modeling and simulation of horizontal vibrations for high-speed elevator, Journal of Shanghai Jiaotong University, 41 (4), 557-560, (2007). https://dx.doi.org/10.3321/j.issn:10062467.2007.04.010

12 Jia Ai-qin, Chen Jian-jun, and Xu Ya-lan. Convex model analysis of vibration control eigenvalues of vehicle suspension system based on perturbation method, Journal of Central South University (Science and Technology), 43 (4), 1320-1324, (2012). http://www.zndxzk.com.cn/down/upfile/soft/20120419/20p1320-91851.pdf

13 Wang Shi-long, Tian Bo, Zhao Yu, et al. Improved shock load model of stranded wires helical springs based on perturbation method, Journal of Mechanical Engineering, 51 (7), 85-90, (2015). https://dx.doi.org/10.3901/JME.2015.07.085

${ }^{14}$ Hou Gong-yu, LI Jing-jing, Zhao Wei-wei, et al. Perturbation solutions for elasto-plastic problems of circular tunnel under unequal compression, Chinese Journal of Rock Mechanics and Engineering, 33 (2), 3639-3647, (2014). https://dx.doi.org/10.13722/j.cnki.jrme.2014.s2.031

15 Guo Zhi-gang and Sun Zhi. Modal analysis of EulerBernoulli beam with multiple open cracks based on perturbation method, Journal of Vibration And Shock, 32 (10), 1-6, (2013). https://dx.doi.org/10.3969/j.issn.10003835.2013.10.001

${ }^{16}$ Niu Ming-taom Li Chang-sheng, and Chen Li-yuan. Perturbation methods for structural-acoustic coupled system with interval parameters, Journal of Vibration And Shock, 34 (10), 194-198, (2015). https://dx.doi.org/10.13465/j.cnki.jvs.2015.10.034

17 Zhang Yi-min Liu Qiao-ling, and Wen Bang-chun. Response analysis of multi-degree-of-freedom nonlinear vibration systems with random parameters of probability perturbation finite element method, Chinese Journal of Computational Mechancs, 20 (1), 8-11, (2003). https://dx.doi.org/10.3969/j.issn.1007-4708.2003.01.003

${ }^{18}$ Fang De-ping and Wang Quan-feng. Accuracy analysis of modified Wilson-theta method of acceleration, Journal of Vibration And Shock, 29 (6), 216-218, (2010). https://dx.doi.org/10.3969/j.issn.1000-3835.2010.06.048 